\title{
INCONTINENCE OF URINE IN WOMEN
}

\author{
By E. CATHERINE LEWIS, M.S., F.R.C.S.
} (Surgeon to the Royal Free Hospital; Senior Surgeon and Urologist to the South London Hospital.)

Few disabilities are so uncomfortable to a woman as urinary incontinence. She is constantly aware of the characteristic odour, is unable to keep her clothes fresh and is much troubled by vulval soreness or pruritus consequent upon the trickling urine. The sufferer becomes selfconscious and disinclined to mix with others or to go to places of amusement, and life becomes a burden to her. Successful treatment is an inestimable boon.

\section{TYPES OF INCONTINENCE}

I. False incontinence. In this condition, which might equally well be termed imperious micturition, the urine is forced out through the normal channel by a sudden contraction on the part of the bladder musculature sufficiently forcible to overcome the resistance of a normal sphincter; it is one of the variable symptoms of the so-called "irritable bladder." The patient experiences a feeling of urgency which becomes momentarily uncontrollable and a small amount of urine escapes. As, however, she is conscious of the spasm, she is usually able to arrest the flow by voluntary contraction of the perineal muscles.

The detrusor mechanism is stimulated to over-action in various ways:-simple congestion of the mucous membrane, acute cystitis or advanced tuberculous lesions, and, in the latter instance the condition is aggravated by a reduced capacity of the bladder. Trigonitis can also be very irritating; in this curious condition where the inflammation, often very acute, is localised to the trigone, the remainder of the bladder appearing perfectly normal, an urge is felt again immediately after micturition and the patient is often actually conscious of the urine trickling down from the ureters over the sensitive surface. In these cases the urine is clear, without deposit, and sterile on culture, though in some it contains a few exfoliated cells, leucocytes and erythrocytes. The solitary ulcer of elderly women, besides being extremely painful, often causes bouts of vesical spasm, as does a malignant growth, particularly the ulcerative variety. The mucosa and consequently the muscle may likewise be irritated by the bladder contentshighly acid or concentrated urine, a foreign body or a stone, or the excitability may accompany other pelvic spasms. Irritability occasionally persists after prolonged pressure on the bladder by fibroids or other pelvic masses even when these have been removed, also after an attack of cystitis cured in all other respects; this is a very tiresome type and difficult to relieve. We must also include here those cases where the incontinence is a neurasthenic or hysterical manifestation, again very refractory to treatment It will be observed that many of these conditions are, or should be, transient and that a normal habit can be regained, but if the trouble is permitted to continue over a long period the bladder may become so inflamed and contracted or otherwise irritable as to be completely intolerant so that a false incontinence merges into the true variety.

2. In True incontinence the sufferer is aware that urine is escaping but, except perhaps in "stress" incontinence, is unable to prevent this by voluntary action, and there is no associated pain apart from such as may be due to soreness and excoriation of the vulva and inner aspect of the thighs. The leakage may be intermittent, partial or total

A. Intermittent or "Stress" incontinence. With this disability the leakage occurs when the intra-abdominal pressure is suddenly raised by any exertion, by a cough or a sneeze or sometimes when the patient rises from a sitting position; in more marked degrees even when she walks about. She is, however, undisturbed at night and when quiescent. As a rule the loss is small, although it may be enough to soak the patient's clothes, but in any event the bladder does not empty itself completely. The cause here is a weakening and stretching of the tissues which normally support the urethra and bladder neck and, in the majority of cases, it follows parturition. The trouble, however, is also met with in elderly or debilitated non-parous women and in persons who have strained themselves by overwork or by lifting heavy weights. A medium degree of pressure by pelvic tumours may have the same effect. This type of incontinence tends to become progressively worse and it preys on the patient's mind and eventually becomes an obsession; but she does know what behaviour on her part is likely to precipitate it and so is able in some measure to guard against the accident.

$B$. Partial true incontinence. The clinical picture in this type is that, though urine dribbles 
away continuously by night as well as by day, the patient micturates normally at more or less regular intervals. Certain cases will be met with where this syndrome has been present from birth and was detected as soon as normal habits in the child should have been established. This is almost certainly due to the deformity known as ectopic ureter where one of these orifices opens on to the vulva or into the vagina and is therefore outside the control of the sphincter mechanism; the urine passing down from the corresponding kidney drips away all the time while the other ureter with a correctly situated orifice fills the bladder in the normal way. The next possibility to be considered is the presence of a fistula of which three varieties may connect the urinary tract with the vagina. They are:-(I) a fistulous ureter, which behaves in the same way as an ectopic one; (2) a vesico-vaginal fistula, which presents this picture if it is so small that the fluid escaping through it does not equal in volume the combined output of the ureters into the bladder; (3) a urethro-vaginal fistula, if this involves the external sphincter, or compressor urethrae muscle, but not the internal sphincter. The two first may follow a parturition injury or a misadventure during a pelvic or perineal operation, also radium treatment of a cervical carcinoma if insufficient care has been taken to protect the neighbouring viscera by means of packing. Partial incontinence from a urethral fistula is more likely to be consequent on an operation as injury to this passage during childbirth, when it does occur, is apt to be more extensive. Other causes are displacement or distortion of the sphincters by par-urethral cysts or tumours, and pressure on the bladder by pelvic, usually malignant, tumours; in the latter case the incontinence is progressive in degree and eventually becomes total.

C. Total continuous incontinence. As the heading implies the urine in this variety drips away all the time and the bladder never fills at all. Certain causes for this will be readily apparent, such as ectopia vesicae and malformations of the urethra where the bladder sphincter does not exist. But this muscle can be defective for other reasons, namely, from destruction during a difficult delivery or from rupture by instrumentation or the insertion of a large object; it may also be completely incompetent as the result of paralysis of its nerve supply or of peripheral neuritis, e.g. in a diabetic patient. Large vesico-vaginal fistulae must also be included here. If treatment is being contemplated it should be borne in mind that a bladder which is kept permanently empty in this way over a long period of time undergoes a shrinkage of disuse and may be very difficult to manage if a repair operation is undertaken.

D. Intermittent total incontinence. This is the distressing condition in which childhood enuresis persists into adult life and the bladder suddenly empties itself completely without any premonitory symptom; actually it is an abnormal act of micturition. Usually when grown-up women are afflicted in this way the accident does not happen so often as with children, and there may be some predisposing cause such as a state of mental excitement or stress, but this is not always the case. It has been known also to occur regularly for two or three nights immediately preceding menstruation; presumably the balance between extrusion and control is naturally unstable in the particular individual and is upset by the increased pelvic congestion.

$E$. Incontinence due to spinal cord disease or injuries may be mentioned but hardly comes within the scope of this paper.

\section{DIAGNOSIS}

History. In the first instance a history of the case must be carefully taken with the particular object of identifying the type of incontinence from which the patient suffers. Thus an obvious deformity can be discovered at once. The questions to be asked will come readily to mind on consideration of the preceding paragraphs.

Clinical examination. With the patient in the dorsal recumbent position the abdomen is inspected and palpated with a view to discovering renal or vesical abnormalities or a mass arising from the pelvis. Next comes examination of the perineum and, to facilitate this, the patient should flex the thighs and separate the knees; it is essential to have a good light focussed on the vulva. Vaginal palpation may reveal a pelvic mass not demonstrable from the abdomen, and any irregularity of surface should be noted as it may indicate the situation of a fistula. Par-urethral cysts or tumours will also be discovered now. After this the vulva is carefully dried and the examiner pauses to watch for any sign of escaping urine. If such is seen the parts must be dried again and an endeavour made to observe where it is coming from. (Should there be any doubt as to whether the fluid really is urine an intravenous injection- $5 \mathrm{cc}$. - of a $0.4 \%$ indigocarmine solution may be given; a blue colouration of the urine should follow in four or five minutes. It must be remembered, however, that the kidney drained by an ectopic or a 
damaged ureter may be poor in function and the test, consequently, not reliable.) If urine is trickling from the vulva or vagina and if no cause has so far been found we think at once of an ectopic ureter or of one of the different kinds of fistulae. If, on the other hand, urine is running from the urethra there may be incompetence of the sphincters from one cause or another, or pressure by pelvic tumours (probably already discovered by palpation). A catheter should now. be passed to ascertain whether or no the leakage is a total one. If the vulva remains dry after being wiped it is clear that the urine is not running away continuously, and the patient is now: asked to cough. It may then perhaps be seen that the anterior vaginal wall beneath the urethra is forced downwards while the external meatus becomes displaced forwards and upwards, and that a small gush of urine escapes; stress incontinence is present. The surgeon now places two fingers one on each side of and parallel with the urethra, and presses upwards, rendering the tissues taut beneath the passage. The patient is asked to cough again; if there is no further escape the indication is that a plastic repair operation will succeed in curing the trouble.

Urine. A specimen of urine, if obtainable, is taken by catheter for full examination, and it must be remembered in this connection that even a so-called "washed-up" specimen from a woman is never entirely reliable, as one drop of vaginal discharge is sufficient to contaminate it and to give a misleading impression.

By this time the cause of the trouble may be clear or at any rate suspected.

Cystoscopy. It is highly desirable that this should be performed although it will be impossible if the urine is leaking away at such a rate that the bladder cannot be distended. Such an examination will show whether the ureteric orifices are present in their normal position, and will demonstrate at once any change in the bladder wall, the presence of a growth or foreign body or any decrease in distensibility of the organ; a fistulous opening is readily seen. If no lesion has been made out and the bladder appears normal the state of the ureters requires investigation, and the dye test already described may be employed to show whether the kidneys are acting equally and well. Should appearance of the dye be delayed or absent on one side a ureteric catheter should be inserted; it will not pass up a fistulous ureter.

Pyelography. If one of the ureteric orifices is absent or if it is found impossible to catheterise one or both of them excretion pyelography is carried out; this is the method in which a preparation which will be excreted by the kidneys in sufficient concentration as to be radioopaque is injected into a vein. In the course of its passage the medium collects to a certain extent in the renal calyces and pelves so that they become visible on an X-ray film; sometimes the ureters can be seen also but not always if they are normal. The first film is taken five minutes after the injection when normal kidneys will have excreted enough of the dye to show, and subsequent ones usually after fifteen, thirty and sixty minutes. Later ones may or may not be called for according to what has already been found. It will be observed that this method of investigation not only demonstrates the size and shape of the hollow portion of the kidneys, but also gives a very fair idea of the renal function. A fistulous ureter is nearly always partially obstructed so that both it and the kidney above it are dilated. It is unlikely that direct, or retrograde, pyelography will be required in investigating a case of urinary incontinence. logist.

Examination of the nervous system, if indicated, calls for the co-operation of a neuro-

\section{TREATMENT}

In false incontinence. As this is a secondary condition the means to be adopted will depend on the underlying cause; thus, the removal of a foreign body, cyst or growth, the administration of antiseptics to combat an infection, an alteration of the state of the urine, and treatment of external conditions all have their place. If the patient is suffering from trigonitis it is a good plan to dilate the urethra and to follow this with a series of instillations of collosol silver; the urine should be kept alkaline and vitamin C. administered. If these methods fail relief may be obtained in some cases by light diathermy fulguration of the trigonal mucosa, an ounce of sedative oil, such as oil of gomenol 5 or 1o \% being left in the bladder. This last is also the method of choice in cases of solitary bladder ulcer and the relief experienced is usually dramatic for the time. The patient should be warned, however, that both trigonitis and solitary ulcer tend to recur and that a permanent cure cannot be promised. The most difficult type of case to deal with is that in which the bladder has become contracted, as the result either of inflammation or of disease, and is mechanically incapable of holding more than a small amount. The neurasthenic and neuralgic bladder is also apt to be refractory. If vesical contraction is due to estab- 
lished tuberculous cystitis no active measures are permissible and palliation only may be attempted. One of the antispasmodic preparations containing belladonna should be given, also sodium bromide in doses of up to 30 grains; the oily instillations already mentioned are very soothing, and it may be noted here that liquid paraffin can be used if other preparations are not available as the effect is obtained partly by coating the ulcerated mucosa. If the contracture is not of tuberculous origin gradual dilatation of the bladder may be tried, also radiant heat and in some cases electrical treatments. When the problem is a "nervous bladder" one method after another usually has to be tried and treatment is likely to be prolonged. It must be of a sedative nature so that again we turn to soothing instillations, applications of warmth such as hot sitz-baths and vaginal douches, perhaps short-wave diathermy, and the administration of alkalies, sodium bromide in large doses, antispasmodics and mild analgesic drugs, also general tonic treatment; curiously enough urethral dilatation sometimes helps these patients. In any case the clinician must be prepared to ring the changes. If some condition is present which cannot be relieved the patient may ultimately have to wear a portable urinal, an appliance which, however well made, is uncomfortable to a woman for anatomical reasons. It does, however, relieve her from the misery of wearing pads which become soaked immediately and cause dermatitis and excoriation of the perineum.

Stress incontinence, when due to weakness of the sub-urethral tissues, can in most cases be cured by a plastic re-inforcing operation. This satisfactory procedure should always be undertaken if the trouble is not of recent origin and provided that the test described under "Diagnosis" promises success. In a case, however, where the weakness follows immediately after parturition in a young woman electrical treatment should be tried; it often effects a cure if begun soon after the injury and is preferable to performing plastic work which may be undone by subsequent child-bearing.

Fistulae. The treatment here is surgical, but it is generally agreed that operative repair of those opening from the bladder into the vagina is a difficult and often disappointing procedure, sometimes requiring several attempts. The outlook depends on the size of the defect, on its position and on its original cause. It is hardly necessary to say that repair of a large opening is very difficult, involving as it does wide undercutting to separate the two organs and to prevent tension when the edges of the hole in the bladder are drawn together; if there is any dragging at the end of the operation the stitches will inevitably cut out to some extent. It is best to warn the patient at the beginning that, although the defect may be reduced in size by a first approach, more than one operation will almost certainly be necessary. With regard to position, a fistula on the base offers more hope of early success than does one on the trigone or inter-ureteric bar owing to the normal fixation of these tissues to the vagina, the close proximity of the ureters and the consequent difficulty of mobilisation. Again a large fistula may be adherent to the pubic bone. Fixed rules cannot be made but, generally speaking, it is best to approach a fistula on the bladder base by suprapubic cystotomy, and one on the trigone from the vagina. As to the cause, fistulae due to radium burns are exceptionally difficult to mend as there appears to be no life or healing power left in the tissues; even if success seems assured in the early stages a fresh break-down may easily occur later, so a very cautious prognosis must be given. In these cases or where the destruction is extensive, perhaps combined with bone fixation, it is better to make no local attempt but to transplant the ureters into the pelvic colon, thus excluding the bladder and turning the rectum into a reservoir for urine as well as for faeces. If an ectopic ureteric opening or a uretero-vaginal fistula exists the ureter should be re-implanted into the bladder unless the corresponding kidney is markedly hydronephrotic, in which case nephrectomy should be performed provided that the second kidney is healthy. Surgical repair is also undertaken for a urethral fistula and the sphincter, if injured, is repaired, though this again may be difficult. If the sphincter has been totally destroyed or is paralysed from failure of the nerve supply ureteric implantation into the colon is the only way to obtain relief.

Congenital deformities of the bladder and urethra are treated by plastic operations designed to meet the particular requirements of the case.

Enuresis in adults is a condition for which no really satisfactory treatment has yet been discovered. In the pre-menstrual type an alteration of the internal secretion balance may be tried and sometimes helps, but where the incontinence occurs at any time and for no apparent reason the clinician can only attempt one remedy after another and is very lucky if any measure of success is attained. Ephedrine in quarter-grain tablets three times daily with an extra one at bedtime has sometimes been found helpful. 